\title{
INTERVALS OF ELECTROHYDRODYNAMIC RAYLEIGH-TAYLOR INSTABILITY: EFFECT OF A NORMAL PERIODIC FIELD
}

\author{
BY \\ ELSAYED F. ELSHEHAWEY * \\ United Arab Emirates University, Al-Ain. United Arab Emirates
}

\begin{abstract}
The intervals of electrohydrodynamic Rayleigh-Taylor instability influenced by a periodic normal field are considered. It is shown that a linear model of the interface is governed by Hill's differential equation. Characteristic values and intervals of stability are discussed. The special case of Mathieu differential equation type is obtained.
\end{abstract}

1. Introduction. Problems of electrohydrodynamic stability have been considered by many authors. (See Melcher [1, 2], Woodson and Melcher [3], Mohamed and Elshehawey [4, 5, 6], Elshehawey [7], Elshehawey et al. [8], El Dabe et al. [9], Mohamed et al. [10,11], and references cited there.) There are some physical situations where one needs a limited band of wave numbers to achieve instability, and at the same time for values of wave numbers less or greater than this band stability is required. For example, in biophysics [12], the cell membrane is formed by a number of adjacent cells if they are subjected to a periodic field. Also the membrane breaks down if a field at a given strength is applied to it. Also, if a force varying periodically with time acts on a mass in such a manner that the force tends to move the mass back into a position of equilibrium in proportion to the dislocation of the mass, one might expect the mass to be confined to a neighborhood of the position of equilibrium. In particular, once the force is strong enough to achieve this effect, one would expect a stronger force to be even more efficient for this purpose. An increase of the restraining force may cause the mass to oscillate with wider and wider amplitudes. The theory of the intervals of instability provides the precise description of this phenomenon.

In the present paper, we shall confine ourselves to giving a general description about the so-called regions of absolute stability, since in most of the previous cases the results were based on numerical computations.

Received January 12, 1989.

* On leave of absence from the Department of Mathematics, Faculty of Education. Ain Shams University, Heliopolis, Cairo, Egypt. 
2. Formulation of the problem. Consider two semi-infinite dielectric inviscid fluids separated by the plane $y=0$. The upper and lower densities of the fluids are $\rho^{(2)}$ and $\rho^{(1)}$, respectively. The fluids are influenced by a periodic electric field

$$
\mathbf{E}_{0}^{(2),(1)}=E^{*} E_{0}^{(2),(1)}(t) \mathbf{e}_{y}, \quad \int_{0}^{\pi} E_{0}^{(1)}(t) E_{0}^{(2)}(t) d t=0,
$$

where $\mathbf{e}_{y}$ is the unit vector in the $y$-direction.

We shall consider all "functions $E_{0}^{(1)}(t) E_{0}^{(2)}(t)$ of class $P$ " which are defined by

$$
\left[\pi \int_{0}^{\pi}\left|E_{0}^{(1)}(t) E_{0}^{(2)}(t)\right|^{p} d t\right]^{1 / p}=1
$$

where $p=1,2,3, \ldots$, or $p=\infty$. If $p=\infty,(2.2)$ means that

$$
\max \left|E_{0}^{(1)}(t) E_{0}^{(2)}(t)\right|=1 .
$$

We assume that $E_{0}^{(1)}(t) E_{0}^{(2)}(t)$ is continuous except for a finite number of points where $E_{0}^{(1)}(t) E_{0}^{(2)}(t)$ may have a jump. We dimensionalise the various quantities using the characteristic length $L=\left(T / \rho^{(1)} g\right)^{1 / 2}$ and the characteristic time $(L / g)^{1 / 2}$.

The motion considered here is irrotational and there exists a velocity potential $\varphi$ such that $\mathbf{v}=\nabla \varphi$.

The velocity potential $\varphi$ satisfies the equation

$$
\frac{\partial^{2} \varphi^{(2) \cdot(1)}}{\partial x^{2}}+\frac{\partial^{2} \varphi^{(2) \cdot(1)}}{\partial y^{2}}=0
$$

such that

$$
\begin{aligned}
& \left|\nabla \varphi^{(2)}\right| \rightarrow 0 \quad \text { as } y \rightarrow \infty, \\
& \left|\nabla \varphi^{(1)}\right| \rightarrow 0 \quad \text { as } y \rightarrow-\infty .
\end{aligned}
$$

The surface deflection is expressed as

$$
y=\xi(x, t),
$$

and

$$
\mathbf{n}=\frac{\nabla F}{[\nabla F \cdot \nabla F]^{1 / 2}}=-\xi_{x}\left(\xi_{x}^{2}+1\right)^{-1 / 2} \mathbf{e}_{x}+\left(\xi_{x}^{2}+1\right)^{-1 / 2} \mathbf{e}_{y},
$$

is the unit normal $\mathbf{n}$ to the surface and $F=0$ is the equation to the surface of separation.

The condition that the interface is moving with the fluid leads to

$$
\xi_{t}-\varphi_{y}^{(2),(1)}+\varphi_{x}^{(2),(1)} \xi_{x}=0 \text { at } y=\xi .
$$

We assume that the quasi-static approximation is valid and we introduce the electrostatic potential $\Psi^{(2),(1)}$ such that

$$
\mathbf{E}^{(2),(1)}=E^{*} E_{0}^{(2),(1)}(t) \mathbf{e}_{y}-\nabla \Psi^{(2),(1)} .
$$

Therefore the differential equation satisfied by $\Psi^{(2),(1)}$ is the Laplace equation

$$
\frac{\partial^{2} \Psi^{(2),(1)}}{\partial x^{2}}+\frac{\partial^{2} \Psi^{(2),(1)}}{\partial y^{2}}=0
$$


along with the following boundary conditions:

(a) The tangential component of the electric field is continuous at the interface

$$
\mathbf{n} \wedge\left(\mathbf{E}^{(2)}-\mathbf{E}^{(1)}=0 \quad \text { at } y=\xi,\right.
$$

which leads to

$$
\xi_{x}\left(\Psi_{y}^{(2)}-\Psi_{y}^{(1)}\right)+\left(\Psi_{x}^{(2)}-\Psi_{x}^{(1)}\right)=\xi_{x} E^{*}\left(E_{0}^{(2)}-E_{0}^{(1)}\right) \text { at } y=\xi .
$$

(b) The normal electric displacement is continuous at the interface $y=\xi(x, t)$ :

$$
\mathbf{n} \cdot\left(\widetilde{\varepsilon}^{(2)} \mathbf{E}^{(2)}-\widetilde{\varepsilon}^{(1)} \mathbf{E}^{(1)}\right)=0 \text { at } y=\xi,
$$

and hence,

$$
\xi_{x}\left(\widetilde{\varepsilon}^{(2)} \Psi_{x}^{(2)}-\widetilde{\varepsilon}^{(1)} \Psi_{x}^{(1)}\right)-\left(\widetilde{\varepsilon}^{(2)} \Psi_{y}^{(2)}-\widetilde{\varepsilon}^{(1)} \Psi_{y}^{(1)}\right)=0, \quad \text { at } y=\xi,
$$

where $\widetilde{\varepsilon}^{(2),(1)}$ is the dielectric constant.

(c) The normal hydrodynamic stress is balanced by the normal electric stress. The balance condition is then [2]

$$
\begin{aligned}
\varphi_{t}^{(1)}- & \rho \varphi_{t}^{(2)}+(1-\rho) \xi+\frac{1}{2}\left(\varphi_{x}^{(1)^{2}}-\rho \varphi_{x}^{(2)^{2}}\right)+\frac{1}{2}\left(\varphi_{y}^{(1)^{2}}-\rho \varphi_{y}^{(2)^{2}}\right) \\
= & \xi_{x x}\left(1+\xi_{x}^{2}\right)^{-3 / 2}-E^{*}\left(\widetilde{\varepsilon}^{(2)} E_{0}^{(2)}(t) \psi_{y}^{(2)}-\widetilde{\varepsilon}^{(1)} E_{0}^{(1)}(t) \psi_{y}^{(1)}\right) \\
& -\frac{1}{2}\left(\widetilde{\varepsilon}^{(2)} \Psi_{x}^{(2)^{2}}-\widetilde{\varepsilon}^{(1)} \Psi_{x}^{(1)^{2}}\right)+\frac{1}{2}\left(\widetilde{\varepsilon}^{(2)} \Psi_{y}^{(2)^{2}}-\widetilde{\varepsilon}^{(1)} \Psi_{y}^{(1)^{2}}\right) \\
& +2 \xi_{x} E^{*}\left(\widetilde{\varepsilon}^{(2)} E_{0}^{(2)}(t) \psi_{x}^{(2)}-\widetilde{\varepsilon}^{(1)} E_{0}^{(1)}(t) \psi_{x}^{(1)}\right) \\
& -2 \xi_{x}\left(\widetilde{\varepsilon}^{(2)} \Psi_{x}^{(2)} \Psi_{y}^{(2)}-\widetilde{\varepsilon}^{(1)} \Psi_{x}^{(1)} \Psi_{y}^{(1)}\right) \\
& -\xi_{x}^{2} E^{*^{2}}\left(\widetilde{\varepsilon}^{(2)} E_{0}^{(2)^{2}}(t)-\widetilde{\varepsilon}^{(1)} E_{0}^{(1)^{2}}(t)\right)+2 E^{*} \xi_{x}^{2}\left(\widetilde{\varepsilon}^{(2)} E_{0}^{(2)}(t) \psi_{y}^{(2)}-\widetilde{\varepsilon}^{(1)} E_{0}^{(1)}(t) \psi_{y}^{(1)}\right) \\
& +\xi_{x}^{2}\left(\widetilde{\varepsilon}^{(2)} \Psi_{x}^{(2)^{2}}-\widetilde{\varepsilon}^{(1)} \Psi_{x}^{(1)^{2}}\right)-\xi_{x}^{2}\left(\widetilde{\varepsilon}^{(2)} \Psi_{y}^{(2)^{2}}-\widetilde{\varepsilon}^{(1)} \Psi_{y}^{(1)^{2}}\right) \\
& -2 \xi_{x}^{3} E^{*}\left(\widetilde{\varepsilon}^{(2)} E_{0}^{(2)}(t) \Psi_{x}^{(2)}-\widetilde{\varepsilon}^{(1)} E_{0}^{(1)}(t) \Psi_{x}^{(1)}\right) \\
& +2 \xi_{x}^{3}\left(\widetilde{\varepsilon}^{(2)} \Psi_{x}^{(2)} \Psi_{y}^{(2)}-\widetilde{\varepsilon}^{(1)} \Psi_{x}^{(1)} \Psi_{y}^{(1)}\right)+O\left(\xi_{x}^{4}\right), \quad \text { at } y=\xi(x, t) .
\end{aligned}
$$

Equations (2.4)-(2.16) will be solved using the method of multiple scale [13].

We introduce the scales $X_{n}$ and $T_{n}$ defined by

$$
X_{n}=\varepsilon^{n} x, \quad T_{n}=\varepsilon^{n} t .
$$

We may also expand $\xi, \Psi^{(2),(1)}$ and $\varphi^{(2),(1)}$ in the form

$$
\begin{gathered}
\xi(x, t)=\sum_{n=1}^{3} \varepsilon^{n} \xi_{n}\left(X_{0}, X_{1}, X_{2} ; T_{0}, T_{1}, T_{2}\right)+O\left(\varepsilon^{4}\right), \\
\Psi^{(2),(1)}(x, y, t)=\sum_{n=1}^{3} \varepsilon^{n} \Psi_{n}^{(2),(1)}\left(X_{0}, X_{1}, X_{2} ; y ; T_{0}, T_{1}, T_{2}\right)+O\left(\varepsilon^{4}\right), \\
\varphi^{(2),(1)}(x, y, t)=\sum_{n=1}^{3} \varepsilon^{n} \varphi_{n}^{(2),(1)}\left(X_{0}, X_{1}, X_{2} ; y ; T_{0}, T_{1}, T_{2}\right)+O\left(\varepsilon^{4}\right),
\end{gathered}
$$


substituting from Eqs. (2.18)-(2.20) into (2.4)-(2.16) and equating coefficients of like powers of $\varepsilon$.

The problem considered here is of the intervals of the linear electrohydrodynamic stability of a single interface stressed by a normal periodic electric field. The effect of nonlinearity on the problem at hand will not be discussed here and it will be the subject of a subsequent paper.

The solutions of the first order problem for travelling waves with respect to the variable $X_{0}$ that decays far from the interface are:

$$
\begin{gathered}
\xi_{1}=D\left(X_{1}, X_{2} ; t\right) e^{i k X_{0}}+\bar{D}\left(X_{1}, X_{2} ; t\right) e^{-i k X_{0}}, \\
\Psi_{1}^{(2)}=\frac{E^{*} E_{0}^{(2)}(t)\left(\widetilde{\varepsilon}^{(2)}-\widetilde{\varepsilon}^{(1)}\right)}{\left(\widetilde{\varepsilon}^{(2)}+\widetilde{\varepsilon}^{(1)}\right)}\left[-D\left(X_{1}, X_{2} ; t\right) e^{i k X_{0}-k y}-\bar{D} e^{-i k X_{0}-k y}\right], \\
\Psi_{1}^{(1)}=\frac{E^{*} E_{0}^{(1)}(t)\left(\widetilde{\varepsilon}^{(2)}-\widetilde{\varepsilon}^{(1)}\right)}{\left(\widetilde{\varepsilon}^{(2)}+\widetilde{\varepsilon}^{(1)}\right)}\left[D\left(X_{1}, X_{2} ; t\right) e^{i k X_{0}+k y}+\bar{D} e^{-i k X_{0}+k y}\right], \\
\varphi_{1}^{(2)}=-\frac{1}{k} \frac{\partial D}{\partial t} e^{i k X_{0}-k y}-\frac{1}{k} \frac{\partial \bar{D}}{\partial t} e^{-i k X_{0}-k y}, \\
\varphi_{1}^{(1)}=\frac{1}{k} \frac{\partial D}{\partial t} e^{i k X_{0}+k y}+\frac{1}{k} \frac{\partial \bar{D}}{\partial t} e^{-i k X_{0}+k y}, \\
\frac{\partial^{2} D}{\partial t^{2}}+\frac{k}{(1+\rho)}\left[1-\rho+k^{2}-\frac{k E^{* 2} E_{0}^{(1)}(t) E_{0}^{(2)}(t)\left(\widetilde{\varepsilon}^{(2)}-\widetilde{\varepsilon}^{(1)}\right)^{2}}{\left(\widetilde{\varepsilon}^{(2)}+\widetilde{\varepsilon}^{(1)}\right)}\right] D=0 .
\end{gathered}
$$

As a special case of Eq. (2.26), the well-known dispersion relation, if we replace the periodic electric field by a constant field, we get the same result given by [4] for the linear system, where

$$
K_{c}=\sqrt{\rho-1}\left(\cosh \theta_{E}+\sinh \theta_{E}\right), \quad \sinh \theta_{E}=\frac{\alpha_{E}}{2 \sqrt{\rho-1}}
$$

and

$$
\alpha_{E}=\frac{E^{* 2}\left(\widetilde{\varepsilon}^{(2)}-\widetilde{\varepsilon}^{(1)}\right)^{2}}{\left(\widetilde{\varepsilon}^{(2)}+\widetilde{\varepsilon}^{(1)}\right)} .
$$

3. Hill's equation. We put Eq. (2.26) in the standard form

$$
\frac{\partial^{2} D}{\partial t^{2}}+[\lambda+Q(t)] D=0
$$

where,

$$
\begin{gathered}
\lambda=\frac{k}{(1+\rho)}\left(1-\rho+k^{2}\right), \\
Q(t)=\beta E_{0}^{(1)}(t) E_{0}^{(2)}(t), \\
\beta=-\frac{k^{2} E^{* 2}\left(\widetilde{\varepsilon}^{(2)}-\tilde{\varepsilon}^{(1)}\right)^{2}}{(1+\rho)\left(\widetilde{\varepsilon}^{(2)}+\widetilde{\varepsilon}^{(1)}\right)}, \quad Q(t+\pi)=Q(t),
\end{gathered}
$$

where $\lambda$ is a parameter depending on ratio density $\rho$ and wave number $k$. Here, $\beta<0$ and $Q(t)$ is a real periodic function of $t$ with period $\pi$. 
Here, we determine the values of $\lambda$ for which the solutions of Hill's equation (3.1) are stable. (See $[14,15,16,17]$ and references cited there.) Following the methods of Magnus and Winkler [16], one can show that, for the given Hill's equation (3.1) there belong two monotonically increasing infinite sequences of real numbers

$$
\lambda_{0}, \lambda_{1}, \lambda_{2}, \ldots,
$$

and

$$
\lambda_{1}^{\prime}, \lambda_{2}^{\prime}, \lambda_{3}^{\prime}, \lambda_{4}^{\prime}, \ldots,
$$

such that Eq. (3.1) has a solution of period $\pi$ iff $\lambda=\lambda_{n}, n=0,1,2, \ldots$, and a solution of period $2 \pi$ iff $\lambda=\lambda_{n}^{\prime}, n=1,2,3, \ldots$.

The $\lambda_{n}$ and $\lambda_{n}^{\prime}$ satisfy the inequalities

$$
\lambda_{0}<\lambda_{1}^{\prime} \leq \lambda_{2}^{\prime}<\lambda_{1} \leq \lambda_{2}<\lambda_{3}^{\prime} \leq \lambda_{4}^{\prime}<\lambda_{3} \leq \lambda_{4}<\cdots
$$

and the relations

$$
\lim _{n \rightarrow \infty} \lambda_{n}^{-1}=0, \quad \lim _{n \rightarrow \infty}\left(\lambda_{n}^{\prime}\right)^{-1}=0 .
$$

The solutions of (3.1) are stable in the intervals

$$
\left(\lambda_{0}, \lambda_{1}^{\prime}\right),\left(\lambda_{2}^{\prime}, \lambda_{1}\right),\left(\lambda_{2}, \lambda_{3}^{\prime}\right),\left(\lambda_{4}^{\prime}, \lambda_{3}\right), \ldots
$$

At the end points of these intervals the solutions of (3.1) are, in general, unstable. This is always true for $\lambda=\lambda_{0}$. The solutions of (3.1) are stable for $\lambda=\lambda_{2 n+1}$ or $\lambda=\lambda_{2 n+2}$ iff $\lambda_{2 n+1}=\lambda_{2 n+2}$, and they are stable for $\lambda=\lambda_{2 n+1}^{\prime}$ or $\lambda=\lambda_{2 n+2}^{\prime \prime}$ iff $\lambda_{2 n+1}^{\prime}=\lambda_{2 n+2}^{\prime}$. For complex values of $\lambda$, Eq. (3.1) always has unstable solutions and it cannot happen here (see Eq. (3.2)).

The $\lambda_{n}$ are the roots of the equation $\Delta(\lambda)=2$ and the $\lambda_{n}^{\prime}$ are those of $\Delta(\lambda)=-2$, where

$$
\Delta(\lambda)=D_{1}(\pi, \lambda)+D_{2}^{\prime}(\pi, \lambda) .
$$

The interval of instability $\left(-\infty, \lambda_{0}\right)$ will always be present (called the zeroth interval of instability), and we define $\left(\lambda_{1}^{\prime}, \lambda_{2}^{\prime}\right)$ as the first interval of instability.

We observe that neither an interval of stability nor an interval of instability can ever shrink to a point. The intervals of stability can never disappear, but two of them can combine to a single one if $\lambda_{2 n+1}=\lambda_{2 n+2}$ or $\lambda_{2 n+1}^{\prime}=\lambda_{2 n+2}^{\prime}$. However, the intervals of instability (with the exception of the zeroth interval) may disappear altogether. This takes place if $Q(t)=\beta E_{0}^{(2)}(t) E_{0}^{(1)}(t)$ is a constant (i.e., for the case of the constant normal electric field given by [4]).

A region in the real $\lambda, \beta$ plane will be called a region of absolute stability for functions of class $p$, if, for any point in this region, (3.1) will have stable solutions for all functions $Q(t)=\beta E_{0}^{(2)}(t) E_{0}^{(1)}(t)$ where $E_{0}^{(2)}(t) E_{0}^{(1)}(t)$ belongs to the class $p$. 
Let $n=0,1,2, \ldots$. The region of absolute stability for functions $E_{0}^{(2)}(t) E_{0}^{(1)}(t)$ of class one is bounded by the curves

$$
\begin{gathered}
\beta_{n+1}= \pm \frac{4(n+1) \sqrt{\lambda}}{\pi} \operatorname{ctg}\left[\frac{\pi \sqrt{\lambda}}{2(n+1)}\right], \quad n^{2}<\lambda<(n+1)^{2}, \\
\beta_{n}= \pm 2 \lambda\left(1-\frac{n}{\sqrt{\lambda}}\right), \quad \lambda>1, n \geq 1, \\
\left.\lambda=0 \quad \text { for } n=0, \text { (i.e., } \rho=1+k^{2}\right),
\end{gathered}
$$

and is such that none of these curves is contained in its interior.

The open region bounded by these curves is maximal; for any point outside or on the boundary of this region, there exists a function $E_{0}^{(2)}(t) E_{0}^{(1)}(t)$ of class one such that not all solutions of (3.1) are bounded. Also, let $m$ be a real variable, $0 \leq m^{2}<1$, and let

$$
M=\int_{0}^{\pi / 2} \frac{d s}{\sqrt{1-m^{2} \sin ^{2} s}}, \quad E=\int_{0}^{\pi / 2} \sqrt{1-m^{2} \sin ^{2} s} d s .
$$

Then the curves defined for $n=0,1,2, \ldots$, by

$$
\begin{gathered}
\beta_{n+1}= \pm 8.3^{-1 / 2} \pi^{-2}(n+1)^{2} M\left[M^{2}\left(m^{2}-1\right)+2 M E\left(2-m^{2}\right)-3 E^{2}\right]^{1 / 2}, \\
\lambda_{n+1}=4 \pi^{-2}(n+1)^{2}\left[M^{2}\left(m^{2}-1\right)+2 M E\right], \quad \lambda>0,
\end{gathered}
$$

bound the region of absolute stability of the functions of class two. The boundary points do not belong to the region, since for

$$
\begin{aligned}
\lambda+\beta E_{0}^{(2)}(t) E_{0}^{(1)}(t)= & 4 \pi^{-2}(n+1)^{2} M^{2}\left(1+m^{2}\right) \\
& -8 \pi^{-2}(n+1)^{2} m^{2} M^{2} \operatorname{sn}^{2}(2(n+1) M t / \pi),
\end{aligned}
$$

the differential equation (3.1) has only one periodic solution (and, therefore, at least one unbounded solution).

The periodic solution (with period $\pi$ or $2 \pi$ ) is

$$
D_{p}=\operatorname{sn} \tau, \quad \tau=2(n+1) M t / \pi,
$$

where $\operatorname{sn} \tau$ is the Jacobian elliptic function with module $m$ and period $4 M$.

Also, for the functions of class $\infty$, the region of absolute stability is bounded by the curves

$$
\begin{aligned}
\left(\lambda_{n+1}\right. & \left.+\beta_{n+1}\right)^{1 / 2} \operatorname{tg}\left[\pi \sqrt{\lambda_{n+1}+\beta_{n+1}} / 4(n+1)\right] \\
= & \left(\lambda_{n+1}-\beta_{n+1}\right)^{1 / 2} \operatorname{ctg}\left[\pi \sqrt{\lambda_{n+1}-\beta_{n+1}} / 4(n+1)\right],
\end{aligned}
$$

where $n=0,1,2, \ldots$, and where the region does not contain any one of these curves in its interior. If one of the square roots should be imaginary, the functions $\operatorname{tg}$ and $\mathrm{ctg}$ have to be replaced by the corresponding hyperbolic functions, i.e.,

$$
E^{* 4}>\frac{\left(1-\rho+k^{2}\right)^{2}\left(\widetilde{\varepsilon}^{(2)}+\tilde{\varepsilon}^{(1)}\right)^{2}}{k^{2}\left(\widetilde{\varepsilon}^{(2)}-\tilde{\varepsilon}^{(1)}\right)^{4}} .
$$

Also, if $a$ and $b$ are real numbers and

$$
a^{2} \leq \lambda+\beta E_{0}^{(2)}(t) E_{0}^{(1)}(t) \leq b^{2},
$$


then the solutions of $(3.1)$ will be stable for all possible $\lambda+\beta E_{0}^{(2)}(t) E_{0}^{(1)}(t)$ satisfying this condition if and only if the interval $\left(a^{2}, b^{2}\right)$ does not contain the square of an integer.

4. Mathieu equation. If we take $\lambda=k /(1+\rho)\left(1-\rho+k^{2}\right)$ as (3.2) and

$$
\begin{gathered}
E_{0}^{(2)}(t) E_{0}^{(1)}(t)=\cos 2 t, \\
q=\frac{+k^{2} E^{* 2}\left(\widetilde{\varepsilon}^{(2)}-\widetilde{\varepsilon}^{(1)}\right)^{2}}{2(1+\rho)\left(\widetilde{\varepsilon}^{(2)}+\widetilde{\varepsilon}^{(1)}\right)},
\end{gathered}
$$

Eq. (3.1) becomes

$$
\frac{\partial^{2} D}{\partial t^{2}}+[\lambda-2 q \cos 2 t] D=0,
$$

which is the Mathieu differential equation [18].

According to Floquet's theorem [17], the general periodic solution of the Mathieu differential equation given by (4.3) can be written as

$$
D_{p}\left(X_{1}, X_{2}, t\right)=F_{1}\left(X_{1}, X_{2},\right) e^{\mu t} H(t)+F_{2}\left(X_{1}, X_{2}\right) e^{-\mu t} H(-t),
$$

where $H(t)$ is a periodic function in $t$ of period $2 \pi$ or $\pi, F_{1}\left(X_{1}, X_{2}\right), F_{2}\left(X_{1}, X_{2}\right)$ are arbitrary constants and $\mu$ is a parameter given by the following relation

$$
\sin ^{2} i \mu \pi=\Delta(0) \sin ^{2} \frac{1}{2} \pi \sqrt{\lambda},
$$

where $\Delta(0)$ is an infinite Hill's determinant depending on $\lambda$ and $q$ (see Ref. [18]), which can take the following form

$$
\Delta(0) \simeq 1-\frac{\pi \lambda^{2} \operatorname{ctg} \frac{1}{2} \pi \lambda^{2}}{4 \sqrt{\lambda}(\lambda-1)} .
$$

It is seen from Eq. (4.4) that if $\mu$ is pure imaginary, the solution for $D_{p}$ will be bounded as $t \rightarrow \infty$ and the system is stable. The characteristic curves of the Mathieu functions and the regions of stability and instability are discussed in [18]. In the $(\lambda, q)$ plane, the regions in which the values of $\lambda$ and $q$ yield imaginary values for $\mu$. These regions are the stable regions [18]. On the other hand, if $\mu$ is real, the solution for $D_{p}$ will tend to $\infty$ as $t \rightarrow \infty$.

The unstable regions (in the plane $(\lambda, q)$ ) are the regions in which the values of $\lambda$ and $q$ correspond to real values of $\mu$. The boundary curves of these regions are symmetric about the $\lambda$ axis. On the other hand, we assume that

$$
q=\frac{+k^{2} E^{* 2}\left(\widetilde{\varepsilon}^{(2)}-\widetilde{\varepsilon}^{(1)}\right)^{2}}{2(1+\rho)\left(\widetilde{\varepsilon}^{(2)}+\widetilde{\varepsilon}^{(1)}\right)}
$$

are small (which is a good approximation to high frequency fields or large wave numbers). Then, following Morse and Feshbach [14], one can show that the solution of Eq. (4.3) will be bounded as $t \rightarrow \infty$ provided that $q$ and $\lambda$ satisfy the following inequality:

$$
4 q^{2}-32(1-\lambda) q+32 \lambda(1-\lambda)>0
$$


or

$$
\frac{k^{4} E^{* 4}\left(\widetilde{\varepsilon}^{(2)}-\widetilde{\varepsilon}^{(1)}\right)^{4}}{(1+\rho)^{2}\left(\widetilde{\varepsilon}^{(2)}-\widetilde{\varepsilon}^{(1)}\right)^{2}}-\frac{16 k^{2} E^{* 2}\left(\widetilde{\varepsilon}^{(2)}-\widetilde{\varepsilon}^{(1)}\right)^{2}(1-\lambda)}{(1+\rho)\left(\widetilde{\varepsilon}^{(2)}+\widetilde{\varepsilon}^{(1)}\right)}+32 \lambda(1-\lambda)>0 .
$$

Also, if $\beta E_{0}^{(2)}(t) E_{0}^{(1)}(t)+\lambda>0$ and

$$
\int_{0}^{\pi}\left[\beta E_{0}^{(2)}(t) E_{0}^{(1)}(t)+\lambda\right]^{2} d t<\left(\frac{64}{\left(3 \pi^{2}\right)}\right)\left\{\int_{0}^{\pi / 2} \frac{d s}{\sqrt{1+\sin ^{2} s}}\right\}^{4}=\frac{1}{12}\left[\frac{\Gamma\left(\frac{1}{4}\right)}{\Gamma\left(\frac{3}{4}\right)}\right]^{4},
$$

then the solutions of Mathieu's equation (4.3) are stable.

Acknowledgments. The author wishes to express his cordial thanks to Professor A. A. Mohamed for helpful discussions.

\section{REFERENCES}

[1] J. R. Melcher, Field Coupled Surface Waves, M.I.T., Cambridge, MA, 1963

[2] J. R. Melcher, Continuum Electromechanics, M.I.T., Cambridge, MA, 1981

[3] H. H. Woodson and J. R. Melcher, Electromechanical Dynamics, part II, John Wiley, New York. 1968

[4] A. A. Mohamed and E. F. Elshehawey, Nonlinear electrohydrodynamic Rayleigh-Taylor instability, part I. A perpendicular field in the absence of surface charges, J. Fluid Mech. 129, 473-494 (1983)

[5] A. A. Mohamed and E. F. Elshehawey, Nonlinear electrohydrodynamic Rayleigh-Taylor instability. II. A perpendicular field producing surface charge, Phys. Fluids 26, 1724, (1983)

[6] A. A. Mohamed and E. F. Elshehawey, Nonlinear electrohyrodynamic Rayleigh-Taylor instability. III. Effect of a tangential field, AJSE 9 (4), 345-360 (1984)

[7] E. F. Elshehawey, Electrohydrodynamic solitons in Kelvin-Helmholtz flow: The case of a normal field in the absence of surface charges, Quart. Appl. Math. 43, 483-501, (1986)

[8] E. F. Elshehawey, Y. O. El Dib, and A. A. Mohamed, Electrohydrodynamic stability of a fluid laver. I. Effect of a tangential field, Nuovo Cimento 1 6D, 291-308 (1985)

[9] N. T. El Dabe, E. F. Elshehawey, G. M. Moatimid, and A. A. Mohamed, Electrohydrodynamic stability of two cylindrical interfaces under the influence of a tangential periodic electric field, $\mathrm{J}$. Math. Phys. 26 (8), 2072-2081 (1985)

[10] A. A. Mohamed, E. F. Elshehawey, and Y. O. El Dib, Electrohydrodynamic stability of a fluid layer. II. Effect of a normal electric field, J. Chem. Phys. 85 (1), 445-455 (1986)

[11] A. A. Mohamed, E. F. Elshehawey, and Y. O. El Dib, Electrohydrodynamic stability of a fluid layer. Effect of a tangential periodic field, Nuovo Cimento 1 8D, 177-192, (1986)

[12] U. Zimmerman, Biochim. Biophys. Acta, 694, 227 (1982)

[13] A. H. Nayfeh, Perturbation Methods Wiley Interscience, New York-London-Sydney, 1973

[14] P. M. Morse and H. Feshbach, Methods of Theoretical Physics, part I, McGraw Hill, 1953

[15] C. M. Bender and S. A. Orszag, Advanced Mathematical Methods for Scientists and Engineers, McGraw Hill, New York, 1978

[16] W. Magnus and S. Winkler, Hill's Equation, Dover, New York, 1979

[17] E. T. Whittaker and G. N. Watson, Modern Analysis, Cambridge, London-New York, 1927

[18] N. W. Mclanchlan, Theory and Applications of the Mathieu Functions, Clarendon Press, Oxford. 1947 